\title{
KINETIC THEORY AND THE KAC MASTER EQUATION
}

\author{
ERIC CARLEN ${ }^{1}$, MARIA C. CARVALHO ${ }^{2}$, AND MICHAEL LOSS ${ }^{3}$
}

\begin{abstract}
This article reviews recent work on the Kac master equation and its low dimensional counterpart, the Kac equation.
\end{abstract}

\section{INTRODUCTION}

As a phenomenological equation, the Boltzmann equation is extremely successful. It is now synonymous with kinetic theory, i.e., the description of a large number of colliding particles. For colliding hard spheres of radius $a$ in a box of volume $V$, it reads

$$
\begin{aligned}
& \frac{\partial}{\partial t} f+v \cdot \nabla_{x} f+F \cdot \nabla_{v} f=\frac{a^{2}}{V^{2}} \int \mathrm{d} w \int_{S^{2}} \mathrm{~d} e|(w-v) \cdot e| \\
\times \quad & {\left[f\left(x, \frac{1}{2}[(v+w)+|v-w| e], t\right) f\left(x, \frac{1}{2}[(v+w)-|v-w| e], t\right)-f(x, v, t) f(x, w, t)\right] }
\end{aligned}
$$

This equation together with the initial conditions $f(x, v, 0)=f_{0}(x, v)$ is a non-linear initial value problem. The function $f(x, v, t)$ is a probability density giving the probability $f(x, v, t) d x d v$ for a particle to have its position and velocity in the cube $d x d v$ centered at $(x, v)$. In many ways, this type of equation can be used to describe any process that evolves under streaming and collisions, which is one of the reasons why this equation is so useful in many different circumstances. Its foundation as the equation of kinetic theory is, however, mysterious. Up to this day, there is no satisfactory derivation of the Boltzmann equation starting from a classical Hamiltonian many body system, notwithstanding the work of Lanford [17, 16, 15]. The problem is that the derivation works only up to the first average collision time. Even in this case, however, the derivation is a mathematical tour de force. Likewise, Illner and Pulvirenti [11, 12 consider the case of a gas jet entering a vacuum in the limit where the number of particles tends to infinity but the particle density is small. Since the gas is expanding in the vacuum the number of collision among the molecules is finite. This allows them to obtain a result for all times.

An attractive feature of the Boltzmann equation is that it allows one to talk in precise terms what is meant by approach to equilibrium. An equilibrium is a stationary solution of the Boltzmann equation. In the absence of an external force $F$, it is a straightforward computation to determine this function to be a global Maxwellian distribution. There is the strong expectation that starting with any initial condition the solution should approach a

E. C. was supported in part by NSF grand DMS-0901632.

M. C. was supported by FCT grant PTDC/MAT/100983/2008.

M. L. was supported in part by NSF grant DMS-0901304. 
global Maxwell distribution for large times. Considerable research has been done on proving return to equilibrium, i.e., studying the long-time behavior of this equation. One of the main tools in this endeavor, the $H$ functional or entropy, has been introduced by Boltzmann himself. Boltzmann proved that $\int f \log f d x d v$ decreases with time. A quantitative version of this result has been obtained by Desvilletes and Villani in [5]. It is, however, a conditional theorem; their result holds for smooth global solutions of the Boltzmann equation. It is unknown whether such solutions exist. The only result in this direction is the one of DiPerna-Lions [7, 8]. These global solutions, however, are weak and not amenable to the analysis in [5].

If one contemplates a bit the times scales between the validity of the Boltzmann equation proved up to now and the desire to understand return to equilibrium, one may wonder what the connection of 'return to equilibrium' and microscopic physics might be. In any case it points to a huge gap in our understanding of the long time evolution of many particle systems.

Since the Boltzmann equation is, for want of a better word, 'paradigmatic' for describing systems consisting of a large number of interacting agents in a probabilistic way, one might ask for a 'derivation', not based on mechanical principles, but based on simple and transparent probabilistic assumptions. This is the path taken by Kac in 1956 [14]. A number of simplifications have to be made. The first is to restrict one's attention to a spatially homogeneous gas, i.e., where the distribution function does not depend on the position, i.e., one considers only the collision terms. This restriction is reasonable since one expects that collisions act on a different time scale than the streaming. In this case the Boltzmann equation takes the form, again written for hard spheres,

$$
\begin{aligned}
& \frac{\partial}{\partial t} f=\frac{a^{2}}{V} \int \mathrm{d} w \int_{\mathbb{S}^{2}} \mathrm{~d} e|(w-v) \cdot e| \\
\times \quad & {\left[f\left(\frac{1}{2}[(v+w)+|v-w| e], t\right) f\left(\frac{1}{2}[(v+w)-|v-w| e], t\right)-f(v, t) f(w, t)\right] . }
\end{aligned}
$$

Thus, one visualizes the times evolution of the full Boltzmann equation as a sequence of collisions interrupted by streaming. This picture is used in the work of Desvilletes and Villani mentioned above and a large part of their work goes into the analysis of the spatially homogeneous Boltzmann equation. Indeed, it presents substantial difficulties concerning the question of approach to equilibrium and as a first step a further simplification can be made by considering Maxwellian molecules in which the collision rate does not depend on the momentum transfer during the collision but just on the angles. The evolution equation then takes the form

$$
\begin{gathered}
\frac{\partial}{\partial t} f=\frac{1}{\tau} \int \mathrm{d} w \int_{\mathbb{S}^{2}} \mathrm{~d} e B\left(\frac{(w-v) \cdot e}{|(w-v)|}\right) \\
\times \quad\left[f\left(\frac{1}{2}[(v+w)+|v-w| e], t\right) f\left(\frac{1}{2}[(v+w)-|v-w| e], t\right)-f(v, t) f(w, t)\right] \\
\int_{\mathbb{S}^{2}} B(w \cdot e) \mathrm{d} e=\frac{1}{2} \int_{-1}^{1} B(x) d x=1 .
\end{gathered}
$$

The form of $B$ is not important but one should remark that for the case where the force law is a repulsive $1 / r^{5}$, the scattering is indeed independent of the momentum transfer and the function $B$ can be expressed in terms of an elliptic function. This was discovered by Maxwell [19. Kac simplified the model further by reducing the problem to one dimensional collisions. Two one dimensional colliding particles either go through each other without changing the momenta, or exchange the momenta. In order not to limit the collision outcomes too much 
one drops the momentum conservation and only retains the energy conservation and considers the Kac equation

$$
\frac{\partial}{\partial t} f=2 \int_{-\pi}^{\pi} \mathrm{d} \theta \rho(\theta) \int_{\mathbb{R}} \mathrm{d} w\left[f\left(v^{\prime}, t\right) f\left(w^{\prime}, t\right)-f(v, t) f(w, t)\right]
$$

where

$$
v^{\prime}=v \cos \theta-w \sin \theta, w^{\prime}=v \sin \theta+w \cos \theta
$$

are the 'post collisional' velocities and where $\rho(\theta) \mathrm{d} \theta$ is a probability measure satisfying

$$
\rho(-\theta)=\rho(\theta) \text {. }
$$

This condition is known as local reversibility since the transitions $(v, w) \rightarrow\left(v^{\prime}, w^{\prime}\right)$ and $\left(v^{\prime}, w^{\prime}\right) \rightarrow(v, w)$ are equally likely. A particularly simple choice is

$$
\rho(\theta)=\frac{1}{2 \pi}
$$

Among all the specializations done so far the really serious ones are the first two, namely passing to the spatially homogeneous case and restricting to Maxwellian Molecules. Most of the results that hold for (3) can be extended to the (21). This non-linear evolution equation can be derived from a master equation as Kac has shown in [14].

The aim of this article is to describe some of the recent results concerning the connections between the equation (3) and the Kac Master equation. Most of these results have been obtained in the last ten years or so. If simple proofs are available they will be presented, otherwise the reader is referred to the literature. In the next section we derive the linear Kac master equation and formulate the question of approach to equilibrium in this new context. In the third section we the connection between the Kac Master equation and Kac's equation (3) will be explained in terms of propagation of chaos. In the fourth section we calculate the gap for the master equation and in the fifth section we talk about approach to equilibrium in the sense of entropy. We end the paper with a number of open problems.

\section{The Kac Master Equation}

Kac's starting point is a random walk in velocity space. Consider $N$ particles moving on the line. As mentioned before, we shall restrict ourselves to the case where the gas is spatially homogeneous. We denote by

$$
\vec{v}=\left(v_{1}, v_{2}, \cdots, v_{N}\right)
$$

the velocity vector, i.e., the coordinate $v_{i}$ is the velocity of the particle carrying the label $i$. The total kinetic energy of this system is

$$
E=\frac{m}{2} \sum_{i=1}^{N} v_{i}^{2}
$$

where we assume that all particles have the same mass $m$. We shall set $m=2$. For the collision law we shall assume that

$$
\left(v_{i}, v_{j}\right) \rightarrow\left(v_{i}^{*}(\theta), v_{j}^{*}(\theta)\right)=\left(\cos \theta v_{i}-\sin \theta v_{j}, \sin \theta v_{i}+\cos \theta v_{j}\right)
$$

where the stared quantities are the post collisional velocities. Clearly, the kinetic energy is preserved. Hence the state space of the system is described by points $\vec{v}$ on the sphere $\mathbb{S}^{N-1}(\sqrt{E})$. In physical collisions the momentum should be conserved too, however, this leads 
to exactly two outcomes: Either the particles go through each other or exchange velocities. Such a collision process would not lead to an ergodic Markov transition operator.

The "Kac walk" can now be described as follows:

(1) Randomly pick a pair $(i, j)$ of distinct indices in $\{1, \ldots, N\}$ uniformly among all such pairs. The particles with labels $i$ and $j$ will collide.

(2) Randomly pick a 'scattering angle' $\theta$ with probability $\rho(\theta) d \theta$ on $[0,2 \pi)$.

(3) Update the velocities by a rotation, i.e.,

$$
\left(v_{i}, v_{j}\right) \rightarrow\left(v_{i}^{*}(\theta), v_{j}^{*}(\theta)\right)=\left(\cos \theta v_{i}-\sin \theta v_{j}, \sin \theta v_{i}+\cos \theta v_{j}\right)
$$

Repeating this process yields a random walk on $\mathbb{S}^{N-1}(\sqrt{E})$.

It is fairly straightforward to construct a Markov transition operator. Let $\vec{v}_{j}$ be the velocities of the particles after the $j$-th collision and let $\phi: \mathbb{S}^{N-1}(\sqrt{E}) \rightarrow \mathbb{R}$ be a continous function. Define the Markov transition operator $Q_{N}$ by

$$
Q_{N} \phi(\vec{v})=\mathbb{E}\left\{\phi\left(\vec{v}_{j+1}\right) \mid \vec{v}_{j}=\vec{v}\right\}
$$

where the expectation is with respect to the probabilities specified above, i.e.

$$
Q_{N} \phi(\vec{v})=\frac{1}{\left(\begin{array}{c}
N \\
2
\end{array}\right)} \sum_{i<j} P_{i, j} \phi(\vec{v})
$$

where

$$
P_{i, j} \phi(\vec{v})=\int_{-\pi}^{\pi} \rho(\theta) \phi\left(R_{i, j, \theta} \vec{v}\right) \mathrm{d} \theta
$$

and

$$
R_{i, j, \theta} \vec{v}=\left(v_{1}, \ldots, v_{i}^{*}(\theta), \ldots, v_{j}^{*}(\theta), \ldots, v_{N}\right) .
$$

Let $F_{0}$ be the initial probability distribution for the velocities $\vec{v}$. The probability distribution $F_{1}$ of the velocities after one collision can be computed via

$$
\int_{\mathbb{S}^{N-1}(\sqrt{E})} \phi(\vec{v}) F_{1}(\vec{v}) \mathrm{d} \sigma^{(N)}=\int_{\mathbb{S}^{N-1}(\sqrt{E})} \mathbb{E}\left\{\phi\left(\vec{v}_{1}\right) \mid \vec{v}_{0}=\vec{v}\right\} F_{0}(\vec{v}) \mathrm{d} \sigma^{(N)}
$$

i.e.,

$$
\int_{\mathbb{S}^{N-1}(\sqrt{E})} \phi(\vec{v}) F_{1}(\vec{v}) \mathrm{d} \sigma^{(N)}=\int_{\mathbb{S}^{N-1}(\sqrt{E})} Q_{N} \phi(\vec{v}) F_{0}(\vec{v}) \mathrm{d} \sigma^{(N)}
$$

Here $\sigma^{(N)}$ is the uniform normalized measure on $\mathbb{S}^{N-1}(\sqrt{E})$. Since $\rho(-\theta)=\rho(\theta)$, the linear operator $Q_{N}$ is selfadjoint on $L^{2}\left(\mathbb{S}^{N-1}(\sqrt{E}), d \sigma^{(N)}\right)$ and since (8) holds for all continuous functions $\phi$, we have that

$$
F_{1}(\vec{v})=Q_{N} F_{0}(\vec{v}) .
$$

Hence $F_{j}$ the probability distribution after $j$ collisions is given by

$$
F_{j}=Q_{N}^{j} F_{0} .
$$

So far, time has not been mentioned, and here we need some further assumptions. It is reasonable to assume that the velocitiy distribution at time $t+d t$ should only depend on the distribution at time $t$ and not on what happened in the past, i.e., we construct a continuous Markov process. Thus, we assume that between time $t$ and $t+d t$ the probability that a collision occurs is

$$
\lambda Q_{N} F(\vec{v}, t) d t+o(d t),
$$


where $\lambda$ is a constant. We assume that the probability that multiple collisions occur in the time interval $[t, t+d t]$ is negligible. Thus, since there are $N$ particles colliding independently, we have, for small time increments,

$$
F(\vec{v}, t+d t)=N \lambda Q_{N} F(\vec{v}, t) d t+(1-N \lambda F(\vec{v}, t)) d t+o(d t),
$$

or

$$
F(\vec{v}, t+d t)-F(\vec{v}, d t)=N \lambda\left[Q_{N} F(\vec{v}, t) d t-F(\vec{v}, t)\right] d t+o(d t) .
$$

Passing to the limit we find the Kac Master Equation

$$
\begin{gathered}
\frac{d}{d t} F(\vec{v}, t)=\lambda N\left[Q_{N}-I\right] F(\vec{v}, t) \\
F(\vec{v}, 0)=F_{0}(\vec{v})
\end{gathered}
$$

where $F_{0}$ is the initial probability distribution.

The initial value problem is now solved by the convergent power series

$$
F(\vec{v}, t)=e^{\lambda N\left(Q_{N}-I\right) t} F_{0}(\vec{v})=\sum_{k=0}^{\infty} \frac{e^{-\lambda N t}(\lambda N t)^{k}}{k !} Q_{N}^{k} F_{0}(\vec{v})
$$

Since $Q_{N}$ is an averaging operator, $Q_{N} 1=1$ and this implies that $F(\vec{v}, t)$ is a probability distribution for all times $t$. A simple computation shows that

$$
\begin{gathered}
\frac{1}{\left(\begin{array}{c}
N \\
2
\end{array}\right)} \sum_{i<j} \int_{-\pi}^{\pi} \rho(\theta) \int_{\mathbb{S}^{N-1}(\sqrt{E})}\left|F\left(R_{i, j, \theta} \vec{v}\right)-F(\vec{v})\right|^{2} \mathrm{~d} \sigma^{(N)} \\
=2\left(\|F\|_{2}^{2}-\left\langle F, Q_{N} F\right\rangle\right)
\end{gathered}
$$

where we denote the inner product on $L^{2}\left(\mathbb{S}^{N-1}(\sqrt{E})\right.$ by $\langle F, G\rangle$. It follows that $Q_{N}$ and hence $e^{\lambda N\left(Q_{N}-I\right) t}$ is ergodic, i.e., $Q_{N} F=F$ only if $F=1$, in particular $Q_{N} \leq 1$. An immediate consequence of this is that $F(\cdot, t)$ converges to the function 1 as time tends to infinity. More precisely, a simple application of the spectral theorem leads to

Lemma 2.1. Assume that $0 \leq F \in L^{2}\left(\mathbb{S}^{N-1}(\sqrt{E}), \mathrm{d} \sigma^{(N)}\right)$ and that

$$
\int_{\mathbb{S}^{N-1}(\sqrt{E})} F(\vec{v}) \mathrm{d} \sigma^{(N)}=1 .
$$

Then we have approach to equilibrium in $L^{2}$, i.e.,

$$
\left\|e^{\lambda N\left(Q_{N}-I\right) t} F_{0}-1\right\|_{2} \rightarrow 0
$$

as $t \rightarrow \infty$.

An issue we explore later is to find rates for the approach to equilibrium. For large systems it is generally observed that the rate of equilibration is independent of the size of the system, i.e., $N$. Thus, the challenge is to find an estimate on the relaxation rate that is uniform in the number of particles.

From now on we will adopt the following conventions. We shall henceforth assume that the function $F_{0}(\vec{v})$ and hence $F(\vec{v}, t)$ is symmetric in the particle labels. We set $\lambda=1$. Finally, since the energy is extensive we can set $E=N$.

With these choices, the Kac master equation reads

$$
\frac{d}{d t} F(\vec{v})=-\mathcal{L} F
$$


with the initial condition

$$
F(\vec{v}, 0)=F_{0}(\vec{v})
$$

where

$$
\mathcal{L}=N\left(I-Q_{N}\right)
$$

and $Q_{N}$ is given by (5), (6) and (7).

\section{Propagation of chaos}

Note that the velocities, viewed as random variables with respect to any probability measure on $\mathbb{S}^{N-1}$ are not independent since

$$
\sum_{j=1}^{N} v_{j}^{2}=N
$$

However, as $N$ gets large one would expect that this dependency gets weaker. The notion of chaos makes this precise. It will be convenient to define it in the language of measure theory. To start we define the marginal measure. Let $\mu^{(N)}$ be a probability measure on $\mathbb{S}^{N-1}(\sqrt{N})$. Fix $k<N$ and pick any Borel $A \subset \mathbb{R}^{k}$. Define

$$
M_{k}\left(\mu^{(N)}\right)[A]=\mu^{(N)}\left[\left\{\left(v_{1}, \ldots, v_{k}\right) \in A\right\}\right],
$$

in other words, we integrate the measure $\mu^{(N)}$ over the sphere but restricting the variables $v_{1}, \ldots, v_{k}$ to remain in the set $A$.

Definition 3.1 (Chaos). Let $\mu$ be a given Borel probability measure on $\mathbb{R}$. A sequence of probability measures $\left\{\mu^{(N)}\right\}_{N=2}^{\infty}$ is called $\mu$-chaotic if each $\mu^{(N)}$ is symmetric under permutations of the particle labels and for each positive integer $k$ the $k$-marginal measure $M_{k}\left(\mu^{(N)}\right)$ converges to $\mu^{\otimes k}$, i.e., for every bounded, continuous test function $\chi\left(v_{1}, \ldots, v_{k}\right)$ we have that

$$
\int \chi\left(v_{1}, \ldots, v_{k}\right) \mathrm{d} \mu^{(N)}\left(v_{1}, \ldots, v_{N}\right) \rightarrow \int \chi\left(v_{1}, \ldots, v_{k}\right) \mathrm{d} \mu\left(v_{1}\right) \cdots \mathrm{d} \mu\left(v_{k}\right)
$$

An illuminating example is given by the Mehler limit 20. In this case the measure $\mu^{(N)}$ is given by the normalized uniform surface measure which is symmetric. Further,

$$
\begin{aligned}
& \int \chi\left(v_{1}, \ldots, v_{k}\right) \mathrm{d} \sigma^{(N)}\left(v_{1}, \ldots, v_{N}\right) \\
= & \frac{\left|\mathbb{S}^{N-1-k}(\sqrt{N})\right|}{\left|\mathbb{S}^{N-1}(\sqrt{N})\right|} \int \chi\left(v_{1}, \ldots, v_{k}\right)\left(1-\frac{\sum_{j=1}^{k} v_{j}^{2}}{N}\right)^{\frac{N-k-2}{2}} \mathrm{~d} v_{1} \cdots \mathrm{d} v_{k} \\
\rightarrow & (2 \pi)^{-\frac{k}{2}} \int \chi\left(v_{1}, \ldots, v_{k}\right) e^{-\frac{\sum_{j=1}^{k} v_{j}^{2}}{2}} \mathrm{~d} v_{1} \cdots \mathrm{d} v_{k}
\end{aligned}
$$

as $N \rightarrow \infty$. For notational convenience we set

$$
\gamma^{(k)}=(2 \pi)^{-\frac{k}{2}} e^{-\frac{\sum_{j=1}^{k} v_{j}^{2}}{2}} .
$$

Let us remark that Kac, in his 1956 paper talked about sequences that have the Boltzmann property, instead of chaotic sequences. In some ways this is a better name and the next theorem makes that clear. 
Theorem 3.2 (Propagation of Chaos, Kac 1956). Let $\left\{F_{0}^{(N)} \sigma^{(N)}\right\}$ be a $f_{0}(v) \mathrm{d} v$-chaotic sequence and denote by $\left\{F_{t}^{(N)} \sigma^{(N)}\right\}$ the sequence of measures where $F_{t}^{(N)}$ is the solution of the master equation, i.e., $F_{t}=e^{N\left(Q_{N}-I\right) t} F_{0}$ for some fixed $t$. Then, $\left\{F_{t}^{(N)} \sigma^{(N)}\right\}$ is a $f(v, t) \mathrm{d} v-$ chaotic sequence and $f(v, t)$ is a solution of the initial value problem

$$
\frac{\partial}{\partial t} f=2 \int_{-\pi}^{\pi} \mathrm{d} \theta \rho(\theta) \int_{\mathbb{R}} \mathrm{d} w\left[f\left(v^{*}(\theta, t) f\left(w^{*}(\theta), t\right)-f(v, t) f(w, t)\right]\right.
$$

with $f(v, 0)=f_{0}(v)$, i.e., it is a solution of the Kac equation. Recall that $v^{*}(\theta)$ and $w^{*}(\theta)$ are given by (4).

For the proof we refer the reader to the original paper of Kac [14].

The above theorem immediately raises the question whether any measure of the form $f(v) d v$ appears as the marginal of a chaotic sequence. Or, more precisely, let $f$ be a probability density on $\mathbb{R}$ with

$$
\int_{\mathbb{R}} v f(v) \mathrm{d} v=0, \int_{\mathbb{R}} v^{2} f(v) \mathrm{d} v=1 .
$$

Is there an $f(v) \mathrm{d} v$ chaotic sequence $\left\{F^{(N)} \sigma^{(N)}\right\}$ ? It is natural to consider $f^{\otimes N}$ which is a function on $\mathbb{R}^{N}$ and to restrict it to the sphere $\mathbb{S}^{N-1}(\sqrt{N})$ and then to control the fluctuations of $\sum_{j=1}^{N} v_{j}^{2}$ with respect to the measure $f^{\otimes N} \mathrm{~d} v_{1} \cdots \mathrm{d} v_{N}$. Since the $v_{i}^{2}$ can be considered as independent random variables with respect to $f^{\otimes N}$, and since the fluctuations are controlled by the second moment of the random variables, this amounts to an assumption on the fourth moment of the function $f(v)$. In fact we have the following theorem [3].

Theorem 3.3 (CCLRV). Let $f$ be a probability density on $\mathbb{R}$ satisfying

$$
\int_{\mathbb{R}} f(v) v^{2} \mathrm{~d} v=1, \int_{\mathbb{R}} f(v) v^{4} \mathrm{~d} v<\infty, f \in L^{\infty}(\mathbb{R})
$$

and let $\mu(\mathrm{d} v)=f(v) \mathrm{d} v$. Then $\left\{\left[\mu^{\otimes N}\right]_{\mathbb{S}^{N-1}(\sqrt{N})}\right.$ is $\mu$-chaotic.

The core of the argument is the following central limit theorem. Set

$$
\Sigma:=\sqrt{\int_{\mathbb{R}}\left(v^{2}-1\right)^{2} f(v) \mathrm{d} v}
$$

and

$$
Z_{N}(f, r):=\int_{\mathbb{S}^{N-1}(r)}\left(\frac{f}{\gamma}\right)^{\otimes N} \mathrm{~d} \sigma_{r}^{(N)}
$$

Theorem 3.4. Assume that

$$
\int_{\mathbb{R}} v f(v) \mathrm{d} v=0, \int_{\mathbb{R}} v^{2} f(v) \mathrm{d} v=1, \int_{\mathbb{R}} f(v) v^{4} \mathrm{~d} v<\infty, f \in L^{p}(\mathbb{R})
$$

for some $p>1$. Then

$$
Z_{N}(f, \sqrt{N})=\frac{\sqrt{2}}{\Sigma}(1+o(1))
$$

as $N \rightarrow \infty$. 


\section{KAC'S CONJECTURE}

Recall that the solution of the Kac Master equation is formally given by $e^{-\mathcal{L}_{N} t}$ where $\mathcal{L}_{N}:=N\left(I-Q_{N}\right)$. One measure of the rate of approach to equilibrium is given by the gap of $\mathcal{L}_{N}$. Define the gap

$$
\Delta_{N}=\inf \left\{\left\langle F, \mathcal{L}_{N} F\right\rangle: F \perp 1,\|F\|_{2}=1\right\} .
$$

If $f$ is any probability distribution that is in $L^{2}\left(\mathbb{S}^{N-1}(\sqrt{N})\right.$, it follows from the spectral theorem that

$$
\left\|e^{-\mathcal{L}_{N} t} f-1\right\|_{2} \leq e^{-\Delta_{N} t}\|f-1\|_{2} .
$$

The important question is whether the gap persists as $N \rightarrow \infty$. In his 1956 paper Kac conjectured that

$$
\Delta_{N} \geq c>0
$$

where $c$ is independent on $N$. After some attempts in [6] this conjecture was proved by E. Jeanvresse [13] using H.-T. Yau's Martingale method. An explicit expression for the gap was computed in [1]. This result was rediscovered in [18]. The following theorem was proved in [1] (see also [2] for more general results).

Theorem 4.1 (CCL). Set $\rho(\theta)=\frac{1}{2 \pi}$. We have that

$$
\Delta_{N}=\frac{1}{2} \frac{N+2}{N-1}
$$

and the gap eigenfunction, unique up to a multiplicative constant, is given by

$$
F_{\Delta_{N}}=\sum_{j=1}^{N}\left(v_{j}^{4}-\frac{3 N}{(N+2)}\right)
$$

It is instructive to compare this result with the gap of the linearized Boltzmann equation. The first marginal of $F_{\Delta_{N}}$ can be easily computed and one obtains

$$
\lim _{N \rightarrow \infty} M_{1} F_{\Delta_{N}}(v)=(2 \pi)^{-1 / 2} e^{-\frac{v^{2}}{2}}\left(v^{4}-6 v^{2}+3\right)=\gamma(v) H_{4}(v) .
$$

To linearize the Kac operator, we set $f=\gamma(v)(1+\varepsilon h)$ and obtain

$$
\frac{1}{\pi} \int_{-\pi}^{\pi} \mathrm{d} \theta \int_{-\infty}^{\infty} \mathrm{d} w \gamma(w)[h(\cos \theta v-\sin \theta w)+h(\sin \theta v+\cos \theta w)-h(v)-h(w)]
$$

The fourth Hermite polynomial is an eigenfunction with eigenvalue $-\frac{1}{2}$ which is the gap of the linearized Kac operator. In fact it is easy to see that all eigenfunctions are given by the Hermite polynomials. (Mc Kean 66 [21], Grünbaum 1972 [10]). Thus, as $N \rightarrow \infty$ all the information one gets from the gap of the Master equation is the gap of the linearized Boltzmann equation. It is likewise easy to see that all the eigenfunctions of the the operator $\mathcal{L}$ are given by spherical harmonics. The tricky part, however, is to decide which among those is the gap eigenfunction.

The proof of the above theorem is not difficult and the method has been useful in a variety of circumstances which gives us the reason to reproduce it here. In a first step one computes

$$
\mathcal{L}_{N} F_{\Delta_{N}}=\frac{1}{2} \frac{N+2}{N-1} F_{\Delta_{N}} .
$$


Hence, $\Delta_{N} \leq \frac{1}{2} \frac{N+2}{N-1}$. The real issue is to prove the reverse inequality. We make an induction argument in the number of particles. For $N=2$

$$
\mathcal{L}_{2}=2\left(I-Q_{2}\right)
$$

and $Q_{2}$ is a one dimensional projection. Hence

$$
\Delta_{2}=2 \text {. }
$$

Write

$$
N\left(I-Q_{N}\right)=\sum_{k=1}^{N}\left(I-Q_{N-1}^{k}\right)
$$

where

$$
Q_{N-1}^{k} F=\frac{1}{\left(\begin{array}{c}
N-1 \\
2
\end{array}\right)} \sum_{i<j, i, j \neq k} P_{i, j} F
$$

i.e., $Q^{k}$ is just the operator acting on the whole space with the interaction with particle $k$ being absent. Thus, for any $F \perp 1$ we have

$$
\left\langle F, N\left(I-Q_{N}\right) F\right\rangle=\sum_{k=1}^{N}\left\langle F,\left(I-Q_{N-1}^{k}\right) F\right\rangle
$$

and we try to use the gap for $N-1$ particles, $\Delta_{N-1}$. Note that for fixed $v_{k}$, the function $F\left(\cdot, v_{k}\right)$ is not perpendicular to the constant function on $\mathbb{S}^{N-2}\left(\sqrt{N-v_{k}^{2}}\right)$. Thus, write $F=$ $\left(F-P_{k} F\right)+P_{k} F$ where $P_{k} F\left(v_{k}\right)$ is the unique function with

$$
\int_{\mathbb{S}^{N-1}(\sqrt{N})} \phi\left(v_{k}\right) F(\vec{v}) \mathrm{d} \sigma^{(N)}=\int_{\mathbb{S}^{N-1}(\sqrt{N})} \phi\left(v_{k}\right) P_{k} F\left(v_{k}\right) \mathrm{d} \sigma^{(N)}
$$

for all test functions $\phi\left(v_{k}\right)$, i.e.,

$$
P_{k} F\left(v_{k}\right)=\int_{\mathbb{S}^{N-2}\left(\sqrt{N-v_{k}^{2}}\right)} F(\vec{v}) d \sigma^{(N-1)}
$$

$P_{k}$, as an operator on $L^{2}\left(\mathbb{S}^{N-1}(\sqrt{N})\right)$, is a selfadjoint projection. Hence

$$
\left\langle F, N\left(I-Q_{N}\right) F\right\rangle=\sum_{k=1}^{N}\left\langle\left(F-P_{k} F\right),\left(I-Q_{N-1}^{k}\right)\left(F-P_{k} F\right)\right\rangle
$$

since $Q_{N-1}^{k} P_{k} F=P_{k} F$. By the induction assumption we know that $\left\langle\left(F-P_{k} F\right),(N-1)\left(I-Q_{N-1}^{k}\right)\left(F-P_{k} F\right)\right\rangle \geq \Delta_{N-1}\left\|\left(F-P_{k} F\right)\right\|^{2}=\Delta_{N-1}\left[\|F\|^{2}-\left\langle F, P_{k} F\right\rangle\right]$, and hence

where

$$
\left\langle F, N\left(I-Q_{N}\right) F\right\rangle \geq \frac{N}{N-1} \Delta_{N-1}\left[\|F\|^{2}-\langle F, P F\rangle\right]
$$

$$
P=\frac{1}{N} \sum_{k=1}^{N} P_{k} .
$$

Setting

$$
\mu_{N}=\sup \{\langle F, P F\rangle: F \perp 1,\|F\|=1\}
$$


we find that

$$
\Delta_{N} \geq \frac{N}{N-1} \Delta_{N-1}\left(1-\mu_{N}\right)
$$

Thus, we need to analyze the operator $P$. To this end we set

$$
\pi_{k}(\vec{v})=v_{k} \quad, \quad \frac{1}{N} \sum_{k=1}^{N} P_{k} F=\mu_{N} F
$$

and notice that for $\mu_{N} \neq 0, F$ is a sum of functions depending only on one variable, i.e., if we set $P_{k} F=h \circ \pi_{k}$

$$
F=\frac{1}{\mu_{N}} \sum_{k=1}^{N} h \circ \pi_{k}
$$

Note that $h$ does not depend on $k$ since $F$ is symmetric. Also note that

$$
\left\langle h \circ \pi_{1}, 1\right\rangle=\left\langle h \circ \pi_{1},\left(\pi_{1}\right)^{2}\right\rangle=0
$$

since

$$
\sum_{k=1}^{N}\left(\pi_{k}\right)^{2}=|\vec{v}|^{2}=N
$$

Now

$$
\frac{1}{N} \sum_{k=1}^{N} P_{1} P_{k} F=\mu_{N} P_{1} F
$$

or

where

$$
\frac{1}{N} h \circ \pi_{1}+\frac{N-1}{N} P_{1} P_{2} F=\mu_{N} h \circ \pi_{1}
$$

$$
P_{1} P_{2} F=:(K h) \circ \pi_{1}
$$

Hence, if we denote by $\kappa_{N}$ the largest eigenvalue of $K$ subject to the conditions

$$
\left\langle h \circ \pi_{1}, 1\right\rangle=\left\langle h \circ \pi_{1},\left(\pi_{1}\right)^{2}\right\rangle=0
$$

then

and

$$
\mu_{N}=\frac{1}{N}+\frac{N-1}{N} \kappa_{N}
$$

$$
\Delta_{N} \geq \frac{N}{N-1} \Delta_{N-1}\left(1-\mu_{N}\right)=\Delta_{N-1}\left(1-\kappa_{N}\right)
$$

Thus, we have reduced the whole problem to the calculation of the spectrum of the operator $K$, which is a relatively simple task. If $h$ is a function on the interval $[-\sqrt{N}, \sqrt{N}]$ then $h \circ \pi_{2}$ as a function on the sphere is constant on the circles of latitude perpendicular to the 2-direction. Now, averaging this function over all rotations that fix the 1-direction delivers a new function which we denote by $(K h) \circ \pi_{1}$. Thus, we have as a quadratic form

$$
\left\langle h \circ \pi_{1},(K h) \circ \pi_{1}\right\rangle=\left\langle h \circ \pi_{1} h \circ \pi_{2}\right\rangle
$$

i.e., the $K$-operator measures the correlation of $h \circ \pi_{1}$ and $h \circ \pi_{2}$ on the sphere. More generally, pick any two unit vectors $\vec{e}_{1}, \vec{e}_{2}$ and consider the form

$$
\left\langle g\left(\vec{v} \cdot \vec{e}_{1}\right) h\left(\vec{v} \cdot \vec{e}_{2}\right)\right\rangle=\left\langle g\left(\vec{v} \cdot \vec{e}_{1}\right)\left(K_{t} h\right)\left(\vec{v} \cdot \vec{e}_{1}\right)\right\rangle
$$


where $t=\vec{e}_{1} \cdot \vec{e}_{2}$. A straightforward calculation yields

$$
\left(K_{t} h\right)(v)=\frac{\left|\mathbb{S}^{N-3}\right|}{\left|\mathbb{S}^{N-2}\right|} \int_{-1}^{1} h\left(v t+\sqrt{N-v^{2}} \sqrt{1-t^{2}} s\right)\left(1-s^{2}\right)^{\frac{N-4}{2}} \mathrm{~d} s .
$$

In our situation $t=0$ and hence we obtain the explicit expression

$$
(K h)(v)=\frac{\left|\mathbb{S}^{N-3}\right|}{\left|\mathbb{S}^{N-2}\right|} \int_{-1}^{1} h\left(\sqrt{N-v^{2}} s\right)\left(1-s^{2}\right)^{\frac{N-4}{2}} \mathrm{~d} s .
$$

The following lemma is proved in [1].

Lemma 4.2. The eigenfunctions of $K$ are polynomials $p_{n}(v)$ with eigenvalues $\alpha_{n}$. The eigenvalues vanish for $n$ odd and

$$
\alpha_{2 m}=(-1)^{k} \frac{\left|\mathbb{S}^{N-3}\right|}{\left|\mathbb{S}^{N-2}\right|} \int_{0}^{\pi}[\cos \theta]^{2 m}[\sin \theta]^{N-3} \mathrm{~d} \theta
$$

In particular

$$
\alpha_{2}=-\frac{1}{N-1}, \alpha_{4}=\frac{3}{N^{2}-1}
$$

$\left|\alpha_{2 m}\right|$ is decreasing in $m$.

The second largest eigenvalue of $P$ is therefore

$$
\Delta_{N} \geq \Delta_{N-1}\left[1-\frac{3}{N^{2}-1}\right]
$$

Since $\Delta_{2}=2$ we easily find

$$
\Delta_{N} \geq \frac{1}{2} \frac{N+2}{N-1}
$$

which proves the theorem.

It is somewhat gratifying that the gap can be computed in other circumstances too. An interesting particular case is where the distribution of the scattering angle is not uniform and given by the density $\rho(\theta)$. It can be easily verified that $F_{\Delta_{N}}$ is always an eigenfunction of $\mathcal{L}_{N}$ with eigenvalue

$$
\Gamma_{N}:=\frac{1}{4} \frac{(N+2)}{N-1} \Gamma_{2}
$$

where

$$
\Gamma_{2}=2 \int_{-\pi}^{\pi}(1-\cos (4 \theta)) \rho(\theta) \mathrm{d} \theta
$$

However, $\Gamma_{N}$ is not the gap in general, since for $N=2$ we may have

$$
\Delta_{2}=2 \min _{k \geq 1} \int_{-\pi}^{\pi}(1-\cos (k \theta)) \rho(\theta) \mathrm{d} \theta<\Gamma_{2}
$$

Our induction scheme applied naively, leads to

$$
\Delta_{N} \geq \frac{\Delta_{2}}{4} \frac{N+2}{N-1}
$$

which proves Kac's conjecture in this case too. We can do better, however. 
Theorem 4.3 (CCL). Assume that

$$
\Delta_{2}>0.45 \Gamma_{2}
$$

then for all $N$ sufficiently large $\Delta_{N}=\Gamma_{N}$ and $F_{\Delta_{N}}$ is the corresponding eigenfunction.

For the proof, which is a bit trickier, we refer the reader to the paper [2]

The Kac Master equation for a spatially homogeneous gas of particles in three dimensional space is analogous to the one dimensional case. Recall that the collision law between two particles is given by

$$
\begin{aligned}
& v_{i}^{*}(w)=\frac{1}{2}\left[\left(v_{i}+v_{j}\right)+\left|v_{i}-v_{j}\right| w\right] \\
& v_{j}^{*}(w)=\frac{1}{2}\left[\left(v_{i}+v_{j}\right)-\left|v_{i}-v_{j}\right| w\right],
\end{aligned}
$$

where $w \in \mathbb{S}^{2}$. These collision preserves now the energy sphere and the momentum plane, i.e., the quantities

$$
\sum_{j=1}^{N}\left|v_{j}\right|^{2}, \text { and } \sum_{j=1}^{N} v_{j}
$$

are preserved. As before, we fix the total energy to be $N$ and, in addition, we fix the total momentum to be zero. The Kac operator is obtained by replacing $P_{i, j}$ in $Q_{N}$ by

$$
P_{i, j} F=\int_{\mathbb{S}^{2}} F\left(v_{1}, \ldots, v_{i}^{*}(w), \ldots, v_{j}^{*}(w), \ldots, v_{N}\right) B\left(\frac{\left(v_{i}-v_{j}\right)}{\left|v_{i}-v_{j}\right|} \cdot w\right) \mathrm{d} w .
$$

It is a selfadjoint operator on the space $L^{2}\left(M, \mu_{N}\right)$ where $M$ is the intersection of the energy sphere with the momentum plane. The measure $\mu_{N}$ is the Euclidean measure on $\mathbb{R}^{3 N}$ restricted to $M$.

It was shown [2] for the case where $B$ is constant, that $\Delta_{N} \geq c>0, c$ independent of $N$. We can, however, say much more. Set

$$
B_{1}=\frac{1}{2} \int_{-1}^{1} x B(x) \mathrm{d} x \text { and } B_{2}=\frac{1}{2} \int_{-1}^{1} x^{2} B(x) \mathrm{d} x
$$

and recall that $\frac{1}{2} \int_{-1}^{1} B(x) \mathrm{d} x=1$. The following two theorems were proved in [4]

Theorem 4.4 (CGL). Suppose that $B_{2}>B_{1}$ and that

$$
\Delta_{2} \geq \frac{20}{9}\left(1-B_{2}\right) \text {. }
$$

Then for all $N \geq 3$

$$
\Delta_{N}=\frac{N}{N-1}\left(1-B_{2}\right)
$$

Moreover, the eigenspace is three dimensional, and is spanned by the functions

$$
\Phi(\vec{v})=\sum_{j=1}^{N}\left|v_{j}\right|^{2} v_{j}^{\alpha}, \alpha=1,2,3
$$


Theorem 4.5 (CGL). Suppose that $\Delta_{2}=2\left(1-B_{1}\right)$. Then for all $N \geq 7$,

$$
\Delta_{N}=\min \left\{\left(1-B_{1}\right), \frac{N}{N-1}\left(1-B_{2}\right)\right\} .
$$

Moreover, if $B_{2}>B_{1}$, the eigenspace is three dimensional, and is spanned by the functions

$$
\Phi(\vec{v})=\sum_{j=1}^{N}\left|v_{j}\right|^{2} v_{j}^{\alpha}, \alpha=1,2,3
$$

If $B_{2}<B_{1}$, the eigenspace is spanned by the functions of the form

$$
\left|v_{i}\right|^{2}-\left|v_{j}\right|^{2} \text { and } v_{i}^{\alpha}-v_{j}^{\alpha}, \alpha=1,2,3
$$

for all $i<j$.

The overall strategy of the proof is the same as in the case for one-dimensional collisions, i.e., the problem is reduced to the study of a low dimensional operator $K$. The eigenvalues, however, do not have any obvious monotonicity properties and the computations are much more complicated. Detailed estimates on Jacobi polynomials are used. We refer the interested reader to [4].

The computations of the gaps for the various models can be considered a success, the drawback is that one learns about as much about the approach to equilibrium as through the linearized Boltzmann equation. More importantly, the notion of gap has other drawbacks and this will be explained in the next section.

\section{ENTROPIC APPROACH TO EQUILIBRIUM}

Using the gap as a rate for approach to equilibrium has an obvious drawback. Assume that $F_{0}=\prod_{j=1}^{N} f_{j}$ subject to the normalization condition

$$
\int_{\mathbb{S}^{N-1}(\sqrt{N})} F_{0}(\vec{v}) \mathrm{d} \sigma^{(N)}=1 .
$$

The almost independence of the functions $f_{j}$ yield that

$$
\left\|F_{0}(\cdot)\right\|_{2} \approx \prod_{j-1}^{N} \int_{\mathbb{S}^{N-1}(\sqrt{N})} f_{j}^{2} \mathrm{~d} \sigma^{(\mathrm{N})}=\mathrm{e}^{\text {const. }} .
$$

Hence, the same is true for $\left\|F_{0}-1\right\|$ and, using the gap estimate only, it will take a time of order $N$ to relax to the equilibrium distribution. The right quantity to consider is Boltzmann's relative entropy

$$
H\left(F \mid \sigma^{(N)}\right):=\int_{\mathbb{S}^{N-1}(\sqrt{N})} F(\vec{v}) \log F(\vec{v}) \mathrm{d} \sigma^{(N)} .
$$

In general if $\mu, \nu$ are two probability measures, their relative entropy is defined by

$$
H(\mu \mid \nu)=\int h \log h d \nu, \quad h=\frac{\mathrm{d} \mu}{\mathrm{d} \nu} .
$$

Thus, if $f(v) \mathrm{d} v$ is a probability measure on $\mathbb{R}$ then the relative entropy of $f \mathrm{dv}$ with respect to the Gaussian function $\gamma(v)$ is given by

$$
H(f \mid \gamma)=\int_{\mathbb{R}} f(v) \log \frac{f(v)}{\gamma(v)} d v
$$


Note that if $F_{N}$ is an $f(v) \mathrm{d} v$ chaotic family one would expect that

$$
H\left(F_{N} \mid \sigma^{(N)}\right)=N H(f \mid \gamma)
$$

as $N \rightarrow \infty$. In other words, the entropy is, like the total energy, an extensive quantity, i.e., proportional to $N$. It has been shown by Boltzmann that $H(f \mid \gamma)$ decreases in time for solutions of the Boltzmann equation. This is the famous H-Theorem. For the Kac equation, this can be readily seen since

$$
\begin{aligned}
& \frac{\mathrm{d}}{\mathrm{dt}} H(f(\cdot, t) \mid \gamma)=\frac{\mathrm{d}}{\mathrm{dt}} \int_{\mathbb{R}} f(v, t) \log f(v, t) \mathrm{d} v-\frac{\mathrm{d}}{\mathrm{dt}} \int_{\mathbb{R}} f(v, t) \log \gamma(v) \mathrm{d} v \\
= & 2 \int_{-\pi}^{\pi} \mathrm{d} \theta \rho(\theta) \int_{\mathbb{R}} \int_{\mathbb{R}}\left[f\left(v^{\prime}, t\right) f\left(w^{\prime}, t\right)-f(v, t) f(w, t)\right] \log f(v, t) \mathrm{d} v \mathrm{~d} w \\
= & \int_{-\pi}^{\pi} \mathrm{d} \theta \rho(\theta) \int_{\mathbb{R}} \int_{\mathbb{R}}\left[f\left(v^{\prime}, t\right) f\left(w^{\prime}, t\right)-f(v, t) f(w, t)\right] \log [f(v, t) f(w, t)] \mathrm{d} v \mathrm{~d} w \\
= & -\int_{-\pi}^{\pi} \mathrm{d} \theta \rho(\theta) \int_{\mathbb{R}} \int_{\mathbb{R}}\left[f\left(v^{\prime}, t\right) f\left(w^{\prime}, t\right)-f(v, t) f(w, t)\right] \\
\times & {\left[\log f\left(v^{\prime}, t\right) f\left(w^{\prime}, t\right)-\log f(v, t) f(w, t)\right] \mathrm{d} v \mathrm{~d} w \leq 0 }
\end{aligned}
$$

Note that we have used the fact that the second moment, the kinetic energy, is preserved in time to drop the term

$$
\frac{\mathrm{d}}{\mathrm{dt}} \int_{\mathbb{R}} f(v, t) \log \gamma(v) \mathrm{d} v
$$

This raises immediately the question for the rates of equilibration [22]. Is there an exponential rate? One could ask the same question for the Kac master equation, i.e., is it true that

$$
\frac{\mathrm{d}}{\mathrm{dt}} H\left(F(\cdot, t) \mid \sigma^{(N)}\right) \leq- \text { const. } H\left(F(\cdot, t) \mid \sigma^{(N)}\right),
$$

for a constant that is, hopefully, independent of $N$. This surmise for the Boltzmann equation is known as Cercigniani's conjecture. The best result so far is by Cedric Villani [22] who proved

Theorem 5.1 (Villani's Theorem). Let $F_{0}$ be any probability density on $\mathbb{S}^{N-1}(\sqrt{N})$ with finite relative entropy $H\left(F_{0}(\cdot) \mid \sigma^{(N)}\right)$. Then the solution of Kac's master equation with initial condition $F_{0}$ satisfies

$$
H\left(F(\cdot, t) \mid \sigma^{(N)}\right) \leq e^{-C_{N} t} H\left(F_{0}(\cdot) \mid \sigma^{(N)}\right)
$$

where

$$
C_{N}=\frac{2}{N-1}
$$

Differentiating $\left.H(F(\cdot, t)) \mid \sigma^{(N)}\right)$ with respect to time yields

$$
\frac{d}{d t} H\left(F(\cdot, t) \mid \sigma^{(N)}\right)=-\int_{\mathbb{S}^{N-1}(\sqrt{N})}\left[\mathcal{L}_{N} F(\vec{v}, t)\right] \log F(\vec{v}, t) \mathrm{d} \sigma^{(N)}
$$

The term

$$
\int_{\mathbb{S}^{N-1}(\sqrt{N})}\left[\mathcal{L}_{N} F(\vec{v})\right] \log F(\vec{v}) \mathrm{d} \sigma^{(N)}
$$


is called the entropy production. Villani proved that for all densities $F$,

$$
\begin{aligned}
& \int_{\mathbb{S}^{N-1}(\sqrt{N})}\left[N\left(I-Q_{N}\right) F(\vec{v})\right] \log F(\vec{v}) \mathrm{d} \sigma^{(N)} \\
\geq & \frac{2}{N-1} \int_{\mathbb{S}^{N-1}(\sqrt{N})} F(\vec{v}) \log F(\vec{v}) \mathrm{d} \sigma^{(N)}
\end{aligned}
$$

that is, the relative entropy production is bounded by $2 /(N-1)$.

Sketch of a proof. The idea is to interpolate densities via the heat kernel on the sphere. Recall that

$$
\Delta=\sum_{i<j} L_{i, j}^{2}
$$

where $L_{i, j}$ is an angular momentum operator, i.e.,

$$
L_{i, j}=v_{i} \partial_{j}-v_{j} \partial_{i}
$$

Set

and compute

$$
F(s)=e^{\Delta s} F
$$

$$
\begin{aligned}
& \frac{\mathrm{d}}{\mathrm{ds}} \int F(s) \log F(s) \mathrm{d} \sigma^{(N)}=\int \Delta F(s) \log F(s) \mathrm{d} \sigma^{(N)} \\
& =-\int \frac{|\nabla F(s)|^{2}}{F(s)} \mathrm{d} \sigma^{(N)}=-4 \int|\nabla \sqrt{F(s)}|^{2} \mathrm{~d} \sigma^{(N)} .
\end{aligned}
$$

Define $\Delta^{k, l}$ by

$$
\Delta=\sum_{i<j} L_{i, j}^{2}=\Delta^{k, l}+L_{k, l}^{2}
$$

Since

we have that

$$
\left[L_{i, j}, \Delta\right]=0 \text { and }\left[\Delta^{k, l}, L_{k, l}\right]=0
$$

$$
e^{\Delta s}=e^{\Delta^{k, l} s} e^{L_{k, l}^{2} s}
$$

and hence

$$
e^{\Delta s} F(x)=e^{\Delta^{k, l} s}\left[e^{L_{k, l}^{2} s} F\right](x)=\int G^{k, l}(x, y ; s)\left[e^{L_{k, l}^{2} s} F\right](y) \mathrm{d} \sigma^{(N)}(y),
$$

where $G^{k, l}(x, y ; s)$ is the kernel associated with the heat semigroup $e^{\Delta^{k, l} s}$. It has the properties

$$
G^{k, l}(x, y, s) \geq 0, \int G^{k, l}(x, y ; s) \mathrm{d} \sigma^{(N)}(y)=1
$$

Set

and note that

$$
F_{k, l}(s)=e^{L_{k, l}^{2} s} F
$$

Since

$$
\lim _{s \rightarrow \infty} F_{k, l}(s)=P_{k, l} F
$$

$$
-4 \int|\nabla \sqrt{F(s)}|^{2} \mathrm{~d} \sigma^{(N)}=-4 \sum_{k<l} \int\left|L_{k, l} \sqrt{F(s)}\right|^{2} \mathrm{~d} \sigma^{(N)} .
$$

and the function

$$
F \rightarrow\left|L_{k, l} \sqrt{F(s)}\right|^{2}
$$


is convex, we can use Jensen's inequality to find the lower bound

$$
\begin{gathered}
-4 \sum_{k<l} \iint G^{k, l}(x, y ; s)\left|L_{k, l} \sqrt{F_{k, l}(y, s)}\right|^{2} \mathrm{~d} \sigma^{(N)}(y) \mathrm{d} \sigma^{(N)}(x) \\
=-4 \sum_{k<l} \iint G^{k, l}(x, y ; s) \mathrm{d} \sigma^{(N)}(x)\left|L_{k, l} \sqrt{F_{k, l}(y, s)}\right|^{2} \mathrm{~d} \sigma^{(N)}(y) \\
=-4 \sum_{k<l} \int\left|L_{k, l} \sqrt{F_{k, l}(y, s)}\right|^{2} \mathrm{~d} \sigma^{(N)}(y) .
\end{gathered}
$$

To summarize, we have shown that

$$
-4 \int|\nabla \sqrt{F(s)}|^{2} \mathrm{~d} \sigma^{(N)} \geq-4 \sum_{k<l} \int\left|L_{k, l} \sqrt{F_{k, l}(y, s)}\right|^{2} \mathrm{~d} \sigma^{(N)}(y)
$$

or

$$
\begin{gathered}
\frac{d}{d s} \int F(s) \log F(s) \mathrm{d} \sigma^{(N)} \geq-4 \sum_{k<l} \int\left|L_{k, l} \sqrt{F_{k, l}(y, s)}\right|^{2} \mathrm{~d} \sigma^{(N)}(y) \\
=-\sum_{k<l} \int \frac{\left|L_{k, l} F_{k, l}(y, s)\right|^{2}}{F_{k, l}(y, s)} \mathrm{d} \sigma^{(N)}(y) \\
=\sum_{k<l} \frac{d}{d s} \int F_{k, l} \log F_{k, l} \mathrm{~d} \sigma^{(N)}
\end{gathered}
$$

Integrating both sides yields

$$
-\int F \log F \mathrm{~d} \sigma^{(N)} \geq \sum_{k<l}\left[\int P_{k, l} F \log P_{k, l} F \mathrm{~d} \sigma^{(N)}-\int F \log F \mathrm{~d} \sigma^{(N)}\right]
$$

or

$$
\begin{gathered}
{\left[\left(\begin{array}{c}
N \\
2
\end{array}\right)-1\right] \int F \log F \mathrm{~d} \sigma^{(N)} \geq \sum_{k<l} \int P_{k, l} F \log P_{k, l} F \mathrm{~d} \sigma^{(N)}} \\
\geq \sum_{k<l} \int P_{k, l} F \log F \mathrm{~d} \sigma^{(N)}=\left(\begin{array}{c}
N \\
2
\end{array}\right) \int Q_{N} F \log F \mathrm{~d} \sigma^{(N)}
\end{gathered}
$$

or

$$
\int\left(I-Q_{N}\right) F \log F \mathrm{~d} \sigma^{(N)} \geq \frac{1}{\left(\begin{array}{c}
N \\
2
\end{array}\right)} \int F \log F \mathrm{~d} \sigma^{(N)}
$$

from which Villani's theorem follows.

Villani's theorem yields an equilibration time that is again of the order $N$, since we have for all densities $F$

$$
\frac{\left\langle\left[\mathcal{L}_{N} F\right], \log F\right\rangle}{H\left(F, \sigma^{(N)}\right)} \geq \frac{2}{N-1} .
$$

This raises the obvious question whether the estimate can be improved. Is there a density $F_{N}$ so that

$$
\lim _{N \rightarrow \infty} \frac{\left\langle\left[\mathcal{L}_{N} F_{N}\right], \log F_{N}\right\rangle}{H\left(F_{N}, \sigma^{(N)}\right)}=0
$$

The following theorem is proved in [3]. 
Theorem 5.2 (CCLRV). For each $c>0$, there is a probability density $f$ on $\mathbb{R}$ with

$$
\int_{\mathbb{R}} v f(v) \mathrm{d} v=0, \int_{\mathbb{R}} v^{2} f(v) \mathrm{d} v=1,
$$

an $f(v) \mathrm{d} v$ chaotic family $\left\{F_{N}\right\}_{N \in \mathbb{N}}$ such that

$$
\limsup _{N \rightarrow \infty} \frac{\left\langle\left[\mathcal{L}_{N} F_{N}(\vec{v}, t)\right], \log F_{N}(\vec{v}, t)\right\rangle}{H\left(F_{N}, \sigma^{(N)}\right)} \leq c .
$$

For each $c$ the density is smooth, bounded and has moments of all order.

If one considers the Kac equation ( 3) instead of the master equation, then the intuition behind this theorem is not difficult to understand. Denote by $\gamma_{a}(v)$ the normalized Gaussian on $\mathbb{R}$ centered at the origin of variance $a$. Now consider the function

$$
f_{\delta}(v)=\delta \gamma_{\frac{1}{2 \delta}}(v)+(1-\delta) \gamma_{\frac{1}{2(1-\delta)}}(v)
$$

where $\delta$ is a small number. The first Gaussian describes an ensemble of a small fraction of particles that have almost all of the kinetic energy, whereas the remaining large fraction of the particles have very little energy. Intuitively, one expects that it would take a long time for such a state to equilibriate. In fact, it is not hard to see that that the entropy production of this state is of order $-\delta \log \delta$, and thus small. With this function $f_{\delta}(v)$ one can construct a $f_{\delta}$ - chaotic state $F_{N}$ in the spirit of Theorem 3.3 which has the properties stated in the previous theorem. Note that, as $\delta$ shrinks to zero, the fourth moment tends to infinity. Villani [22] conjectured that there exists a constant $C$ such that for every $N$ there is $F_{N}$ with

$$
\lim _{N \rightarrow \infty} \frac{\left\langle\left[\mathcal{L}_{N} F_{N}\right], \log F_{N}\right\rangle}{H\left(F_{N}, \sigma^{(N)}\right)}<\frac{C}{N} .
$$

This conjecture was essentially proved recently by Amit Einav [9].

Theorem 5.3. For any $0<\beta<\frac{1}{6}$ there exists a constant $C_{\beta}$ depending only on $\beta$ such that

$$
\Gamma_{N} \leq \frac{C_{\beta} \log N}{N^{1-2 \beta}}
$$

The crux of the matter is to make the statement in Theorem 5.2, the statement about a chaotic state and its marginal quantitative. The key theorem is an analog of Theorem 3.4, which can be rendered in a much stronger form since it is a statement about the particular function $f_{\delta}(v)$.

Theorem 5.4. Let $f_{\delta_{N}}(v)=f_{\delta_{N}}(v)=\delta \gamma_{\frac{1}{2 \delta_{N}}}(v)+\left(1-\delta_{N}\right) \gamma_{\frac{1}{2\left(1-\delta_{N}\right)}}(v)$ where $\delta_{N}$ is chosen such that

$$
\delta_{N}^{1+2 \beta} \cdot N \underset{N \rightarrow \infty}{\longrightarrow} \infty, \delta_{N}^{1+3 \beta} \cdot N \underset{N \rightarrow \infty}{\longrightarrow} 0
$$

Then for a fixed $j$

$$
Z_{N-j}\left(f_{\delta_{N}}, \sqrt{u}\right)=\frac{2}{\sqrt{N-j} \cdot \Sigma_{\delta_{N}} \cdot\left|\mathbb{S}^{N-j-1}\right| u^{\frac{N-j}{2}-1}}\left(\frac{e^{-\frac{(u-N+j)^{2}}{2(N-j) \Sigma_{\delta_{N}}^{2}}}}{\sqrt{2 \pi}}+\lambda_{j}(N-j, u)\right)
$$

where $\sup _{u \in \mathbb{R}}\left|\lambda_{j}(N-j, u)\right| \leq \epsilon_{j}(N)$ and $\lim _{N \rightarrow \infty} \epsilon_{j}(N)=0$. 
Theorem 5.3 or rather its proof is an application of the interplay between the Kac equation and the Kac Master equation. In this particular instance knowing a state of low entropy production for the Kac equation yields a state of low entropy production for the Kac Master equation. This connection can be cast as entropic chaos. Let $\mu$ be a probability measure on $\mathbb{R}$ and for each $N$ let $\mu^{(N)}$ be a probability measure on $S^{N-1}(\sqrt{N})$. The sequence $\left\{\mu^{(N)}\right\}_{N \in \mathbb{N}}$ is said to be entropically $\mu$-chaotic if it is $\mu$-chaotic and in addition

$$
\lim _{N \rightarrow \infty} \frac{H\left(\mu^{(N)} \mid \sigma^{N}\right)}{N}=H(\mu \mid \gamma)
$$

Theorem 5.5. Let $f$ be a probability density on $\mathbb{R}$ satisfying

$$
\int_{\mathbb{R}} f(v) v^{2} \mathrm{~d} v=1, \int_{\mathbb{R}} f(v) v^{4} \mathrm{~d} v<\infty, f \in L^{\infty},
$$

and set $\mu(\mathrm{d} v)=f(v) \mathrm{d} v$. Then $\left\{\left[\mu^{\otimes N}\right]_{\mathbb{S}^{N-1}(\sqrt{N})}\right\}_{N \in \mathbb{N}}$ is entropically $\mu$-chaotic, in fact for any $k \in \mathbb{N}$ we have

$$
\lim _{N \rightarrow \infty} H\left(M_{k}\left(\left[\mu^{\otimes N}\right]_{\mathbb{S}^{N-1}(\sqrt{N})}\right) \mid \mu^{\otimes k}\right)=0
$$

Moreover, let $\left\{\mu^{(N)}\right\}_{N \in \mathbb{N}}$ be any family of symmetric probability measures on $\mathbb{S}^{N-1}(\sqrt{N})$ with

$$
\frac{1}{N} H\left(\mu^{(N)} \mid\left[\mu^{\otimes N}\right]_{\mathbb{S}^{N-1}(\sqrt{N})}\right) \rightarrow 0
$$

Then $\left\{\mu^{(N)}\right\}_{N \in \mathbb{N}}$ is entropically $\mu$-chaotic.

For a proof the reader may consult [2].

It would be nice to know whether or not it is true that for any entropically chaotic family $\left\{\mu^{(N)}\right\}_{N \in \mathbb{N}}$ for any $k$

$$
\lim _{N \rightarrow \infty} H\left(M_{k}\left(\mu^{(N)}\right) \mid \mu^{\otimes k}\right)=0 .
$$

This is an open problem. Using a diagonal argument together with the previous theorem one obtains

Corollary 5.6. Let $f$ be a probability density on $\mathbb{R}$ with

$$
\int_{\mathbb{R}} f(v) v^{2} \mathrm{~d} v=1, H(f \mid \gamma)<\infty .
$$

Then there exists an $f(v) \mathrm{d} v$-entropically chaotic sequence.

While exponential entropic decay in time for general initial conditions is false one may ask for natural conditions on $F_{N}$ such that

$$
\frac{\left\langle\mathcal{L}_{N}, \log F_{N}\right\rangle}{H\left(F_{N} \mid \sigma^{(N)}\right)} \geq c>0
$$

for some $c$ independent on $N$. This problem is completely open. One could weaken the above question by asking whether there are there natural conditions on $f$ so that one can construct a $f$-chaotic sequence $\left\{F_{N}\right\}$ with

$$
\frac{\left\langle\mathcal{L}_{N}, \log F_{N}\right\rangle}{H\left(F_{N} \mid \sigma^{(N)}\right)} \geq c>0
$$

for some $c$ independent on $N$ ? Nothing is known about this problem either. 


\section{REFERENCES}

[1] E. Carlen, M. C. Carvalho, and M. Loss. Many-body aspects of approach to equilibrium. In Séminaire: Équations aux Dérivées Partielles, 2000-2001, Sémin. Équ. Dériv. Partielles, pages Exp. No. XIX, 12. École Polytech., Palaiseau, 2001.

[2] E. A. Carlen, M. C. Carvalho, and M. Loss. Determination of the spectral gap for Kac's master equation and related stochastic evolution. Acta Math., 191(1):1-54, 2003.

[3] Eric A. Carlen, Maria C. Carvalho, Jonathan Le Roux, Michael Loss, and Cédric Villani. Entropy and chaos in the Kac model. Kinet. Relat. Models, 3(1):85-122, 2010.

[4] Eric A. Carlen, Jeffrey S. Geronimo, and Michael Loss. Determination of the spectral gap in the Kac model for physical momentum and energy-conserving collisions. SIAM J. Math. Anal., 40(1):327-364, 2008.

[5] L. Desvillettes and C. Villani. On the trend to global equilibrium for spatially inhomogeneous kinetic systems: the Boltzmann equation. Invent. Math., 159(2):245-316, 2005.

[6] Persi Diaconis and Laurent Saloff-Coste. Bounds for Kac's master equation. Comm. Math. Phys., 209(3):729-755, 2000.

[7] R. J. DiPerna and P.-L. Lions. On the Fokker-Planck-Boltzmann equation. Comm. Math. Phys., 120(1):123, 1988.

[8] R. J. DiPerna and P.-L. Lions. Global solutions of Boltzmann's equation and the entropy inequality. Arch. Rational Mech. Anal., 114(1):47-55, 1991.

[9] Amit Einav. An improved upper bound on the Entropy Production for the Kac Master equation. To appear in Kinetic and Related Models.

[10] F. Alberto Grünbaum. Linearization for the Boltzmann equation. Trans. Amer. Math. Soc., 165:425-449, 1972.

[11] R. Illner and M. Pulvirenti. Global validity of the Boltzmann equation for two- and three-dimensional rare gas in vacuum. Erratum and improved result: "Global validity of the Boltzmann equation for a two-dimensional rare gas in vacuum" [Comm. Math. Phys. 105 (1986), no. 2, 189-203; MR0849204 (88d:82061)] and "Global validity of the Boltzmann equation for a three-dimensional rare gas in vacuum" [ibid. 113 (1987), no. 1, 79-85; MR0918406 (89b:82052)] by Pulvirenti. Comm. Math. Phys., 121(1):143$146,1989$.

[12] Reinhard Illner and Mario Pulvirenti. Global validity of the Boltzmann equation for a two-dimensional rare gas in vacuum. Comm. Math. Phys., 105(2):189-203, 1986.

[13] Elise Janvresse. Spectral gap for Kac's model of Boltzmann equation. Ann. Probab., 29(1):288-304, 2001.

[14] M. Kac. Foundations of kinetic theory. In Proceedings of the Third Berkeley Symposium on Mathematical Statistics and Probability, 1954-1955, vol. III, pages 171-197, Berkeley and Los Angeles, 1956. University of California Press.

[15] Oscar E. Lanford, III. Time evolution of large classical systems. In Dynamical systems, theory and applications (Recontres, Battelle Res. Inst., Seattle, Wash., 1974), pages 1-111. Lecture Notes in Phys., Vol. 38. Springer, Berlin, 1975.

[16] Oscar E. Lanford, III. On a derivation of the Boltzmann equation. In International Conference on Dynamical Systems in Mathematical Physics (Rennes, 1975), pages 117-137. Astérisque, No. 40. Soc. Math. France, Paris, 1976.

[17] Oscar E. Lanford, III. A derivation of the Boltzmann equation from classical mechanics. In Probability (Proc. Sympos. Pure Math., Vol. XXXI, Univ. Illinois, Urbana, Ill., 1976), pages 87-89. Amer. Math. Soc., Providence, R. I., 1977.

[18] David K. Maslen. The eigenvalues of Kac's master equation. Math. Z., 243(2):291-331, 2003.

[19] J. Clerk Maxwell. Matter and motion. Dover Publications Inc., New York, N. Y., undated.

[20] Mehler, F. G., Ueber die Entwicklung einer Function von beliebig vielen Variablen nach Laplaceschen Functionen höherer Ordnung, Journal für die reine und angewandte Mathematik 66: 161-176, 1866.

[21] H. P. McKean, Jr. Speed of approach to equilibrium for Kac's caricature of a Maxwellian gas. Arch. Rational Mech. Anal., 21:343-367, 1966.

[22] Cédric Villani. Cercignani's conjecture is sometimes true and always almost true. Comm. Math. Phys., 234(3):455-490, 2003. 
1 Rutgers University, Department of Mathematics,, CARlen@math.rutgers.ed

E-mail address: carlen@math.rutgers.edu

2 Department of Mathematics and CMAF, University of Lisbon, 1649-003 Lisbon, Portugal E-mail address: mcarvalh@cii.fc.ul.pt

3 Georgia Institute of Technology, School of Mathematics, Atlanta, Georgia 30332-0160, LOSS@MATH.GATECH.EDU

E-mail address: Ioss@math.gatech.edu 